\title{
Untersuchungen über den zeitlichen Faktor der Zellteilung.
}

II. Mitteilung: Über das Wesen nnd das Vorkommen der Determination der Zellteilung.

Von

Dr. Alexander Gurwitsch,

Professor an der Hochschule für Franen.

(Aus dem histologischen Laboratorium der Hochschule für Franen in St. Petersburg.)

Mit 4 Figuren im Text.

Eingegangen am 24. März 1911.

In einer kürzlich in diesem Archiv veröffentlichten Arbeit „Über Zufall, Normierung und Determination in der Ontogenese 1) babe ich den Nachweis zu erbringen versucht, daß die Zellteilungen während der Ontogenese vielfach eine Art Regelung erfahren, die ich als "Normierung " derselben bezeichnet habe.

Es soll letzterer Begriff besagen, daß, falls innerhalb eines größeren, als homogen anzusehenden Zellkomplexes die Entscheidung tiber das Eintreffen oder Ausbleiben der Zellteilung in dieser oder jener Zelle in jedem gegebenen Zeitabschnitte dem Zufall (bei gleichbleibenden Chancen) tiberlassen wäre, das Ergebnis nicht anders ausfiele, als es in zahlreichen, verschiedenartigen Objekten tatsächlich zur Beobachtung gelangt.

Sind die für einen derartigen Vergleich notwendigen Prämissen, deren ausflihrliche Besprechung bei andrer Gelegenheit erfolgen soll, erfüllt, so berechtigt ein derartiges Zusammentreffen zu einem Analogieschluß auf die Art des den Zeitpunkt der Zellteilung bedingenden Faktors. Derselbe ist als \& zufällig * zu bezeichnen, und es kann auf

1) Festachrift f. Wilhelm Roux. Dieses Archiv. Bd. 30. Teil I. 
denselben die in der Statistik bzw. Wahrscheinlichkeitslehre iubliche Betrachtungsweise Anwendung finden.

Es dürfte angebracht sein, die Berechtigung dieser Aufstellung an der Hand des ublichen Urnenschemas ausführlich durchzuführen. Denken wir uns einen aus lauter gleichartigen Zellen bestehenden Komplex, welcher durch eine Symmetrieebene halbierbar ist. Jede Zelle erhält eine Ordnungsnummer, und zwar die Zellen links von der Symmetrieebene ungerade, rechts von der Ebene entsprechende gerade Bezeichnungen. Jede Zelle einer Seite hat demnach einen Partner auf der andern Seite.

Wir nehmen nun aus einer Urne mit gleicher Anzahl schwarzer und weißer Kugeln wiederholte Ziehungen vor; nach jeder Ziehung kommt die Kugel in die Urne zurïck.

Jede weiße Kugel soll das Stattfinden einer Zellteilung, jede schwarze Kugel das Ausbleiben einer solchen bedeuten und die Ordnungsnummer der Ziehung sich auf die entsprechend numerierte Zelle beziehen.

Gehen wir vermittels derartiger Ziehungen sämtliche Zellen unsres Komplexes durch, so erhalten wir ein buntes Verteilungsbild der Teilungsfiguren, welches als Resultat eines rein zufallsmäßigen Geschehens anzusehen ist, wobei bei der gewählten Versuchsanordnung letzteres durch Gleichheit der Chancen für und wider und durch Konstanz derselben während der Versuchsdauer, sc. für den ganzen Zellkomplex charakterisiert wird. Durch entsprechend abgeänderte Füllung der Urne, d. h. in Abhängigkeit von dem Verhältnis von Schwarz zu Weiß, läßt sich natiirlich dasselbe Schema mit beliebig hohen bzw. geringen Chancen zugunsten des Stattfindens der Mitose durchführen.

Die Analyse einer derartigen Verteilung läßt sich von zwei Ausgangspunkten vornehmen. Verfügen wir über die Kenntnis der sog. Wahrscheinlichkeit a priori oder der Ursachen * 1 ), welche dem Geschehen zugrunde liegen, so können wir mit einem angebbaren Grad von Genauigkeit das Ergebnis vorhersagen. In unserm Fall z. B., wenn wir über das Zahlenverhältnis von schwarzen und weißen Kugeln in der Urne orientiert sind und die Sicherheit haben, daß die Ziehungen ohne Kenntnis der Farbe und nach vorhergehendem Durchrühren der Kugeln vorgenommen werden, können wir Angaben a priori über die voraussichtliche Verteilung der Mitosen machen, bzw.

1) Hier sUrsaches in weiteren Sinne der Wahrscheinlichkeitsrechnung begriffen. 
genügende Kriterien gewinnen, nach welchen wir gegebenenfalls eine Verteilung der Fälle als nicht durch reinen Zufall bei gleichbleibenden Chancen oder als unter wechselnden Chancen geschehend erkennen können.

Dieser Schluß ist aber nicht ohne weiteres in seinem ganzen Umfange umkehrbar.

Finden wir eine Verteilung der Fälle (in unserm Falle der Mitosen) innerhalb eines größeren Komplexes, die sich mathematisch als der zufallsmäßigen ganz analog charakterisieren läßt, so kann die Schlußfolgerung auf die tatsächliche Zufälligkeit des bestimmenden Faktors nur dann als genügend gesichert erscheinen, wenn wir die Überzeugung haben, daß die Prämissen der ersteren im Objekte erfüllt sind. In unserm Fall wäre es eine notwendige Voraussetzung, daß der untersuchte Komplex aus lauter gleichartigen Zellen zusammengesetzt ist, daB mit andern Worten für jede Zelle die gleiche Wahrscheinlichkeit a priori für das Eintreffen der Mitose bestehe.

Diese Überzeugung von der gleichen Wahrscheinlichkeit a priori ist selbstverständlich von dem mathematischen Kalkül ganz unabhängig und muB aus der Gesamtheit der Umstände, d. h. aus genügender vorhergehender Kenntnis des Untersuchungsobjekts, gewonnen werden, was ja in unsern Objekten keinerlei Schwierigkeiten bereitet.

Wir können demnach folgenden Satz aufstellen:

Finden wir innerhalb eines Komplexes von sonst ${ }^{1}$ ) gleichartigen Zellen eine Verteilungsweise der Mitosen vor, welche, was sich rechnerisch nachweisen läßt, mit dem Ergebnis des Urnenschemas zusammentrifft, so läßt sich darans folgern, daß für die Bestimmung des Zeitpunktes des Eintreffens einer Mitose, abgesehen von einer für den ganzen Komplex geltenden Chancengebung (dem Verhältnis von schwarzen und weißen Kugeln in der Urne vergleichbar), keinerlei spezielle, furr einzelne Zellen verschiedene, entweder von der Zelle

1) Die Beurteilung der durchgehenden Gleichartigkeit der betreffenden Zellen muß selbstverständlich von verschiedensten Gesichtspunkten ausgehen, und zwar nicht nur die Abstammung, die funktionelle und morphologische, sondern auch, und wohl vor allem, die prospektive Identität derselben berïcksichtigen. In unsern Objekten kann diesen Postulaten meist nur insofern Genüge geschehen, als aus dem Zellkomplex symmetrische Gruppen identischer Zellen herausgesucht werden. Es dürfte dagẹgen nur in den seltensten Fällen der ganze Komplex aus lauter gleichartigen (und unter gleichen Bedingungen stehenden) Zellen bestehen. So können z. B. in der Zwiebelwurzel als durchaus gleichartig die, einer konzentrischen Schicht angehörigen Zellen betrachtet werden usw. 
selbst oder von ihren Beziehungen zur Umgebung (zum Ganzen) ausgehende Faktoren oder Momente vorliegen.

Aufgabe rein biologischer, ev. experimenteller, Forschung bleibt es schließlich, fur den Begriff der $>$ Chancen* einen faßbaren und konkreten Inhalt zu finden.

Das einfachste Schema, nach welchem hier verfahren werden kann, liegt in der Definition des Zufälligen (sc. des Chancenmäßigen) als eines durch Zusammenwirken oder Zusammentreffen mindestens zweier voneinander unabhängiger Ursachenkomplexe bedingten Geschehens.

-Ursache* wird hier im engeren Sinne, d. b. als vorangehende Veränderung, als Prozeß, verstanden und den übrigen, für das Geschehen notwendigen, aber konstanten "Bedingungen * gegenuibergestellt.

Es wird dabei natürlich vorausgesetzt, daß der Ablauf dieser veränderlichen Faktoren in allen als identisch betrachteten Zellen in identischer Weise nomologisch bestimmt wird. Stellen wir uns z. B. diese Vorgänge als Bewegungen vor, so wäre anzunehmen, daß die Gesetzlichkeiten derselben bis auf einen Parameter bestimmt sind. Wir können dabei zum Vergleich etwa die Ätherschwingungen in einem nichtpolarisierten Lichtstrahl heranziehen.

Ein Zusammentreffen bestimmter Phasen derartiger unabhängig voneinander ablaufender, aber in sich streng geregelter Vorgänge trägt den Charakter des Zufalls 1 ).

1) Es ergibt sich aus dieser Definition der Normierung, daß, was ja eigentlich selbstverständlich ist, die causale Erklärung des Zufalls nur als Verschiebung des Problems möglich ist, $\mathrm{da}$ ja die e definitione regellose Variabilität je eines Parameters jedes veränderlichen Faktors, oder, was aufs gleiche hinanskommt, der Beziehungen zwischen den veränderlichen Faktoren, an sich erklärungsbedürftig bleibt. Es muß aber, wie v. KRIES treffend hervorhebt, zwischen nomologischer und ontologischer Kenntnis eines Vorganges streng geschieden werden: >das Wissen.... unvollständig nur in ontologischer Beziehung zu sein braucht, während die Einsicht in den gesetzmäßigen Zusammenhang des Geschehens, in die nomologischen Verhältnisse, vollständig und korrekt sein könnte. In einer kinetischen Theorie der Gase z. B., welche den Molekülen bestimmte Wirkungsgesetze zuschriebe und auf Grund solcher Annahmen alle Erscheinungen befriedigend erklärte, würden wir ein Wissen besitzen, welches unsern wichtigsten und vornehmsten intellektuellen Bedürfnissen genügte. Im Vergleich damit könnten die Fragen nach ontologischen Bestimmungen von lediglich singulärer Bedeutung, die Frage z. B., wie gegenwärtig in einem bestimmten Gase alle Moleküle angeordnet sind und sich bewegen, von untergeordneter Bedeutung seine (v. KrIEs, Die Prinzipien der Wahrscheinlichkeitsrechnung. Freiburg 1886. S. 172). Haben wir es in unserm Fall mit dem Problem des szufälligen. 
Aus der vorangehenden Definition des Begriffes Normierung läßt sich derjenige der Determination ableiten.

Wir wollen zunächst, um unsre Vorstellungen zu fixieren, an unsrer Fiktion der Normierung, d. h. an zwei voneinander unabhängige, in jeder Beziehung bis auf einen Parameter fixierte Bewegungen, festhalten.

Denken wir uns jetzt die eine oder die andre Modifikation dieses ursprlinglichen Verhaltens verwirklicht.

1) Die beiden veränderlichen Faktoren, die im Falle einer Normierung als voneinander unabhängig gedacht werden, seien nunmehr durch irgendeine funktionale Beziehung miteinander in Verbindung gebracht. Es bestehe eine Beziehnng zwischen dem Zustand (bzw. dem Evolutionsstadium) des Faktors $A$ und demjenigen des Faktors $B$.

Ist für das Stattfinden des von $A$ und $B$ abhängigen Prozesses, z. B. in unserm Falle der Zellteilung, ein zeitliches oder räumliches Zusammentreffen des Zustandes $A_{n}$ und $B_{n}$ erforderlich, so ist dasselbe in der Art der funktionalen Verknüpfung der Evolution von $A$ und $B$ bereits im positiven oder negativen Sinne prädestiniert: die Kombination $A_{n} \times B_{n}$ findet im Zeitpunkt $t_{n}$ statt, oder ist überhanpt unmöglich.

2) Es sei von zwei variablen Faktoren $A, B$, der eine Faktor in einem bestimmten Erolutionsstadium konstant geworden. Ist es das Stadium $A_{n}$, welches für das Eintreffen des Vorganges notwendig ist, so muß letzterer in fataler Weise eintreten, sobald der evolutionierende Faktor $B$ das Stadium $B_{n}$ erreicht hat.

Es gehört in diesem Fall eine funktionale Beziehung zwischen dem Zeitpunkt des Konstantwerdens des Faktors $A$ and dem entsprechenden Evolutionsstadium des Faktors $B$ nicht zur Voraussetzung.

3) Der eine der variablen Faktoren sei in seiner Veränderungsweise nicht nur nomologisch, sondern auch ontologisch bestimmt, bzw. kein einziger Parameter desselben, und zwar im Gegensatz zum Verhalten im Falle der Normierung, unbestimmt gelassen. So wäre z. B. eine periodische Bewegung denkbar, deren Phasen letzterenfalls zwar zeitlich, nicht aber räumlich bestimmt wären; wir können hier

Eintreffens eines wichtigen Vorganges, wie die Zellteilung, zu tun, so kann es füglich als Erklärung gelten, wenn wir denselben als Ergebnis des Zusammenwirkens bestimmter nomologisch streng geregelter Abläufe erkennen, mit der einzigen Restriktion, daß wir ïber die ontologische Bestimmung ihrer gegenseitigen Beziehungen nichts aussagen können. 
wiederum zum Vergleich die Art der Ätherschwingungen heranziehen: das Verhalten derselben im nichtpolarisierten Lichte hätte dem Falle der Normierung, die Bedingungen des polarisierten Strahles unserm zweiten Postulate entsprochen.

Es ergibt sich ohne weiteres, daß alle hier fingierte Möglichkeiten einer Verursachung, denen sich noch verschiedene andre anreihen lassen, za Resultaten führen, die von denjenigen der Normierung grundverschieden sind und ibre Bezeichnung als Determination des Geschehens rechtfertigen.

Es lassen aber gleichzeitig diese Konstruktionen eine Brïcke von dem Begriffe der Normierung zu demjenigen der Determination schlagen und entheben uns der großen logischen Schwierigkeit, einen Vorgang unter bestimmten Verhältnissen für *zufällig ", in ganz analogen Fällen dagegen, eventuell beim gleichen Objekt, für streng geregelt zu erklären.

Ich möchte mich an dieser Stelle mit dieser kurzen, rein formell begrifflichen Definition der Determination begnügen, nachdem in meiner ersten Arbeit eine mathematische Definition derselben gegeben wurde. In einer nachfolgenden theoretischen Arbeit werde ich noch Gelegenheit haben, auf die allgemeine Bedentung der Begriffe der Normierung und der Determination zurückzukommen 1).

1) Es dürfte von Interesse sein, eine Nachahmung der Determination vermittels des Urnenschemas durchzuführen, wodurch aber selbstverständlich nur die prinzipielle Analogie, nicht die Verwandtschaft der hier und dort in Betracht kommenden Faktoren gemeint werden soll.

Denken wir uns die vorhergehende Versuchsanordnung durch zwei weitere Urnen ergänzt, und zwar durch eine mit einem Vorwiegen weißer, und eine zweite mit einem entsprechenden Übergewicht schwarzer Kugeln.

Die erste Ziehung, die sich auf die Zelle Nr. 1 bezieht, wird wie vorher aus der Urne mit gleicher Anzahl schwarzer und weißer Kugeln vorgenommen. Je nach dem Ergebnis dieser Ziehung wird aber die zweite aus der zweiten, bzw. der dritten Urne ausgeführt, und zwar mit der Absicht, für die der ersten Zelle (links von der Mittellinie) symmetrische zweite Zelle (rechts von der Mittellinie) dasselbe Ergebnis herbeizuführen. Ergibt demnach die erste Ziehung weiß, so soll die zweite Ziehung aus der Urne mit einem Übergewicht weißer Kugeln, d. h. mit größeren Chancen fuir weiß, vorgenommen werden, und umgekehrt. Die dritten, und im allgemeinen die ungeraden Ziehungen sollen demnach aus der Urne mit gleicher Anzahl schwarz und weiß, die geraden Ziehungen mithin je nach dem Ergebnis der ungeraden aus der zweiten bzw. dritten Urne geschehen.

Es leuchtet ein, daß das Gesamtergebnis einer nach dieser Vorschrift ausgeführten größeren Versuchsreihe eine viel größere Symmetrie in der Verteilung der Teilungsfiguren, eine größere Genanigkeit des Geschehens ergeben wird, ohne daß durch die Versuchsbestimmungen jeder Einzelfall eindeutig bestimmt 
Aus der vorhin gegebenen Definition der Determination ergeben sich folgende Korollare:

a. Es ist logisch berechtigt, auch von negativer Determination zu sprechen, namentlich wenn man die zweite Formulierung der Verursachung in Betracht zieht. Durch einen ev. temporären Stillstand eines variablen Faktors $A$ in einem bestimmten Zustande kann nämlich das Geschehen für eine bestimmte Zeit unmöglich gemacht werden. Daß diese Determination ins Negative unter Umständen etwas andres bedeutet als ein Unvermögen, das Fehlen einer Potenz im betreffenden Objekte, möge durch folgendes Beispiel erörtert werden: Bestimmte Zellarten, namentlich hochorganisierter Gewebe, die sich unter normalen Verhältnissen nicht mehr vermehren, verhalten sich recht verschieden in dieser Hinsicht bei der Regeneration: Ganglienzellen vermögen sich auch in diesem Fall nicht zu teilen, verschiedene andre Zellarten, wie z. B. Leberzellen, vermehren sich unter abnormen Verhältnissen in sehr lebhaftem Tempo.

Haben wir auch vielleicht genügenden Grund zur Annahme, daß den Nervenzellen die Teilungspotenz, der Teilungsmechanismus fehlt, so ist es ungereimt, das gleiche von der Leberzelle anzunehmen, welche die mechanische Läsion eines ev. relativ entfernten Gewebebezirkes durch eine Teilung beantwortet.

Es ist jedenfalls vorsichtiger und sachgemäßer, sich zu denken, daß der Teilungsmechanismus in letzteren Zellen etwa wie ein Uhrschlagwerk »arretiert* ist und durch allerlei geringe Eingriffe in Gang gesetzt werden kann.

Durch "Ingangsetzen * des arretierten Faktors wird die negative Determination in eine s Normierung « des Geschehens verwandeit, wie wir solche z. B. bei der Verteilung der Mitosen in den Regenerationszonen, soviel zu beurteilen ist, tatsächlich auch finden.

wäre. Es ist aber jedes Einzelergebnis nicht mehr als von den übrigen Ergebnissen völlig unabhängig zu bezeichnen, es besteht ein gewisser Grad von Beeinflussung derselben, die in dem vorgeschlagenen Schema, um die Sache nicht zu sehr zu komplizieren, als einseitig gedacht sind, aber natürlich ohne iede Schwierigkeit durch Einfügen weiterer Urnen bzw. Versuchsvorschriften in eine gegenseitige verwandelt werden kann. Der Gegensatz zwischen unserm Normierungsschema und letzterer Versuchsanordnung wird aber erst klar, wenn wir uns überlegen, daß 1000 nachfolgende Ziehungen aus einer Urne in ersten Fall durch simultane Ziehungen ans 1000 gleichzeitig aufgestellten Urnen ersetzt werden dürfen, was die gegenseitige Unabhängigkeit der Einzelfälle ztr Evidenz beweist, wogegen die Voranssetzungen der zweiten Versucbsanordnung eine Aufeinanderfolge der Einzelziehungen unentbebrlich machen. 
Durch *Verstellen * des arretierten Faktors in eine für das Zustandekommen des Geschehens passende Lage, wäre ein Übergang von negativer zur positiven Determination denkbar. Ob diese Möglichkeit je zur Verwirklichung kommt, ist sehr zweifelhaft.

b. Aus dem Absatz 2 folgt, daß die Determination eines Geschehens mit der Genauigkeit desselben nicht als synonym betrachtet werden darf. Setzen wir nämlich voraus, daß der »arretierte c Faktor $A$ in seiner vorangegangenen Evolution von dem Faktor $B$ unabhängig gewesen ist, so wird der Zeitpunkt des Eintreffens des betreffenden Vorganges innerhalb desjenigen Spielraumes schwanken, welcher durch die eventuelle zeitliche Verschiedenheit des Faktors $B$ in den verschiedenen Zellen gegeben ist. Denken wir uns, um uns die Sache klar zu machen, die Variabilität des $B$ als einen periodischen Prozeß mit regelmäßigen Phasen und verlegen wir das für das Zustandekommen des Vorganges notwendige Stadium des $B$ in die Phase $B_{\imath}$. Haben wir es nun mit einer Gruppe identischer Zellen zu tun, in welchen die Arretierung des Faktors $A$ streng simultan geschah, so kann der Zeitpunkt des Eintreffens des Geschehens, in Abhängigkeit von der Phasenverschiedenheit des $B$ in den Zellen, innerhalb des Spielraumes der Periode des $B$ schwanken.

c. Das oben benutzte Urnenschema klärt uns nur über die Verhältnisse der diskontinuierlichen Chancensetzung auf. Denken wir uns das Verhältnis ron $\frac{S}{S+W}$ nahe 1 , so würden wiederholte Ziehungen ein Geschehnis zutage fördern, welches den Anschein einer absoluten Gesetzlichkeit haben könnte, solange als nicht einmal, als große Seltenheit, auch eine weiße Kugel zum Vorschein käme. Dieses seltene Ereignis wäre selbstverständlich genügend, um uns über die wahre Sachlage aufzuklären, ein analoges Verhalten innerhalb eines reellen Kollektivgegenstandes pflegt aber seltsamerweise nicht nach Analogie des Urnenschemas beurteilt zu werden. Man pflegt hier von Ausnahmen zu sprechen, deren Entstehungsgründe ganz abseits von der supponierten strengen Grundgesetzlichkeit liegen und dem Eingreifen zufälliger, störender Momente zu verdanken wären.

Es kommen aber, wie im folgenden nachgewiesen werden soll, Fälle zur Beobachtung, in welchen nach Erwägung aller Umstände eine derartige Auffassung der "Ausnahmen * ganz und gar unzulässig ist, wo mit andern Worten das Geschehen trotz seiner imponierenden Regelmäßigkeit sich als normiert entpuppt und folglich auch ein entsprechendes causales Schema erheischt. 
Es kann und muß demnach, solange wir uns an ein Geschehen mit diskontinuierlicher Chancensetzung halten, zwischen einer Normierung mit fast ausnahmslosem Geschehen und der hier vorgetäuschten Determination streng geschieden werden.

Das Gesagte gilt aber nicht für den Fall kontinuierlicher Wahrseheinlichkeit bzw. Chancensetzung. Denken wir z. B. eine der Ursachen als Bewegung und setzen wir die Wahrscheinlichkeit des Geschehens als stetige (reziproke) Funktion der Geschwindigkeit derselben, so ist es klar, daB hier die Determination als echter Grenzbegriff der Normierung erscheint.

Die vorangehenden Erörterungen über die Determination hatten bezweckt, ein allgemeines begriffliches Schema für eine biologische Interpretation der auf mathematischem Wege in einem Kollektivgeschehen ermittelten Determination zu geben.

Die fingierten variablen Faktoren werden selbstverständlich durch rein biologische Begriffe bzw. Geschehensarten vertreten werden müssen und der vielfach im vorhergehenden zur Anwendung gekommene Vergleich derselben mit verschiedenen Bewegnngsarten soll nur der Anschanlichkeit dienen und ist selbstverständlich eine bloße Fiktion.

Auch hatte die in meiner ersten Arbeit versuchte Identifizierung einer von den bei der Zellteilung tätigen variablen. Faktoren mit Kernbewegungen nur den Wert einer nnverbindlichen Arbeitshypothese.

Die im weiteren mitzuteilenden Ergebnisse sind natiurlich noch unendlich fern von der definitiven Lösung der gestellten Probleme. Sie ermöglichen aber einen tieferen Einblick in das Wesen der Determination der Zellteilung, d. h. derjenigen regulatorischen Bestimmungen des Prozesses, welche denselben in höherem Maße den Interessen des Ganzen unterordnen, als es durch bloße *Chancensetzung *, welche der Normierung zugrunde liegt, erreichbar ist.

Wir kennen mehrere Objekte, in denen die Verteilung der Zellteilungen den Verdacht auf eine Determination derselben erweckt. Als solche seien folgende angefúhrt:

1) Scheitelzellen in den Vegetationspunkten kryptogamer Pflanzen und, analog denselben, Teloblasten in den Mesodermstreifen der Anneliden. 
2) Furchungsteilungen, namentlich der regelmäBigen Furchungstypen.

3) Kernteilungen in mehrkernigen Zellen bzw. Syncytien, die fast ausnahmslos synchrom ablaufen.

4) Spermatogoniale Teilungen bei verschiedenen Wirbellosen und Wirbeltieren, sowie sporogene Teilungen verschiedener Pflanzen.

Die Furchnngsteilungen, namentlich der meisten Wirbellosen, lassen in ihrer verblitffenden Exaktheit das Stattfinden einer Determination ohne weiteres erkennen, bedürfen somit keiner speziellen, daraufhin gerichteten Untersuchung.

Es wurde außerdem bereits in der ersten, oben zitierten Arbeit daranf hingewiesen, daß noch im Gastrulastadium der Echinidenkeime die Verteilung der Mitosen einen deutlich unternormalen Dispersionsgrad aufweist, was ebenfalls auf eine Determination derselben schließen läßt.

Unsre Untersuchung beschränkt sich vorläufig auf die spermatogonialen Teilungen in den Amphibienhoden, die aus verschiedenen Gründen für unsre Probleme besonders geeignet sind.

Die mikroskopische Anatomie des Urodelenhodens wurde namentlich von Meves in erschöpfender Weise geschildert.

Je nach der Jahreszeit finden wir in den cranialen Lappen jedes Hodens versehiedene Generationen der Samenzellen, von den Ursamenzellen angefangen bis anf die Spermatiden vor.

Die Ursamenzellen liegen in größeren Ballen dicht beieinander, wobei jedoch jede Ursamenzelle von einem Kranz von Follicularzellen umgeben ist und sich in der Weiterentwicklung als von ihren Nachbarn völlig unabhängig erweist. Durch wiederholte Teilung einer Ursamenzelle entsteht ein größeres Nest von Spermatogonien (Spermatogemme), welches von der mittlerweile durch die Follicularzellen gebildeten bindegewebigen Kapsel vollständig abgeschlossen bleibt. Innerhalb dieser Kapsel laufen auch sowohl die Rubeteilungen, als die spermatogenetischen Prozesse ab. Jede Kapsel oder Cyste enthält schlieBlich einen dichtgedrängten Büschel von fertigen Spermien.

Eine Anzahl derartiger Nester gruppiert sich schon früheitig um ein gemeinsames Lumen und bildet in ihrer Gesamtheit eine größere, durch stärkere bindegewebige Septa abgeschlossene Cyste, woran sich jedes Nest in Form eines mehr oder weniger regelmäßig geformten Kegels oder Pyramide beteiligt. 
Die streng monophyletische Entstehung jedes Nestes kann als völlig sichergestellt betrachtet werden, da eine allseitig abgeschlossene bindegewebige Kapsel um jedes derselben sich schon fribzeitig erkennen läßt. Sollten im ubrigen $a b$ und zu auch zwei Ursamenzellen in eine gemeinsame Follicularkapsel geraten, so bätte anch dieser Umstand für unsre Untersuchung keine Bedeutung. Ein Zellaustausch zwischen den Nestern erscheint jedoch nach allem als völlig ausgeschlossen.

Der Hauptpunkt, welcher diese Spermatogemmen zu änßerst wichtigen Objekten cytologischer Forschung macht, liegt in der sonst kaum noch erreichten Identität ihrer Einzelelemente.

Es handelt sich nicht nur um die Identität der monophyletischen Herkunft, sondern auch der prospektiven Bedeutung; die hier wohl die prospektiven Potenzen völlig erschöpft und in höchst regelmäßiger Weise zur Spermatogenese, als zum definitiven und scharf abgesetzten Abschluß des Evolutionscyclus derselben führt.

Die Identität der höchst komplizierten spermatogenetischen Prozesse innerhalb jedes Nestes gehört zu den merkwürdigsten Ausnahmserscheinungen des organischen Geschehens, wo wir doch überall ein bestimmtes Variationsmaß als Regel vorfinden.

Es wäre ein vergebliches Beginnen, mit unsern heutigen optischen Mitteln irgendwelche Variationen in der Größe, Konfiguration der Einzelteile der wachsenden Spermien innerbalb eines Nestes zu suchen, wenn wir von einigen Momenten in der gegenseitigen Lagerung der Centrosomen zum hinteren Kernpol auf bestimmten Entwicklungsstadien absehen. Wenn wir aber in Betracht ziehen, daß die komplizierten Umwandlungen, namentlich der Centrosomen, regelmäßig in der Zeit fortschreitend sind, so ist eine völlige Identität ihrer Größe, Form usw. in allen Zellen eines Nestes mit einem geradezu ideellen Synchronismus der betreffenden Prozesse gleichbedeutend.

Der Lebenscyclus der Samenzellen einer Gemme ist demnach nicht nur dem Inhalte, sondern auch der Zeit nach in geradezu ideeller Weise geregelt.

Das die Zellen gewissermaßen verknüpfende Band ihrer Identität kommt nicht nur in ihrem gemeinsamen Ursprung und ihrer Äquifinalität zur Geltung. Ein ganz abseits stehender, den Lebenscyclus der Samenzellen jäh abschließender, durchaus nicht selten auftretender Degenerationsvorgang ergreift ebenfalls stets ganze Zellnester. 
Soweit man sich auf Grund fixierter Präparate von letzterem eine Vorstellung bilden kann, handelt es sich im wesentlichen um eine Verflussigung des Chromatins, welches zunächst lachenartig, dann in Form einer kompakten Calotte den mittlerweile stark angeschwollenen Nucleolns umgibt. Die Kernmembran und die Zellkonturen schwinden schließlich spurlos.

Über die Ursachen dieses Vorganges, welcher in keinem Hoden vermißt wird, sind wir in voller Unkenntnis. Es ist aber für uns vor allem von Wichtigkeit, daß der Eintritt der Degeneration nicht an einen bestimmten Entwicklungspunkt der Spermatogonien geknüpft ist, bzw. in den verschiedensten Generationen derselben erfolgen kann und stets und ausnahmslos alle Zellen eines Nestes gleichzeitig ergreift. Der Synchronismus der Degenerationsstadien in allen Zellen eines Komplexes ist ein höchst vollkommener.

Es können demnach die Zellen eines Spermatogemmas in jeder Hinsicht $u n d$ in jedem Abschnitt ihres Evolutionscyclus als schlechterdings identisch betrachtet werden, wobei das eben geschilderte Verhalten derselben den Degenerationsvorgängen gegenüber eine Identität auch der Gesamtheit ibrer physiologischen und funktionellen Zustände, einschließlich der Tempi der Abläufe, voraussetzen läßt.

Es bietet sich dadurch die kostbare, möglicherweise die einzige Gelegenheit, den Grad der Regelung der Zellteilung an einer größeren Anzahl identischer Objekte zu priffen, bzw. die Beziehungen zwischen letzterem Vorgang und der Gesamtheit der tubrigen Geschehensabläufe in den Zellen zu klären.

Das Problem stellt sich demnach folgendermaßen:

1) Fällt der Zellteilungsvorgang in den als identisch anzusprechenden Zellen eines Nestes ebenso synchron aus, wie es nachweisbar sowohl die Degenerationsprozesse als die Spermiogenese sind?

2) Ist die Gesamtzahl der spermatogonialen Teilungen, bzw. der der Spermatidenbildung vorangehenden Generationen ftur alle Zellen eines Nestes identisch und konstant?

In ihrer Beantwortung erweisen sich beide Fragen eng miteinander verkniipft. Die erste Durchsicht der Schnitte zeigt uns ohne weiteres, daß Mitosen der Samenzellen nie sporadisch anftreten, sondern daß stets große Gruppen gleichzeitig in Teilung begriffen sind. Die erste Frage scheint demnach ohne weiteres im positiven Sinne beantwortet zu sein.

Ein tieferes Studium des Objektes ergibt aber ganz andre und höchst wichtige Verhältnisse. 
Untersuchungen über den zeitlichen Faktor der Zellteilung. II. 459

a. Es werden $a b$ und $z a$, wenn auch sehr selten, Nester vorgefunden (vgl. Tab. S. 462 Abschn. C), in denen neben einer Mehrzahl sich teilender Zellen einzelne oder auch mehrere Ruhezellen liegen.

b. Der Synchronismus der mitotischen Phasen ist nur sehr selten ein annähernd vollkommener. Es werden, zuweilen mehr durcheinander, häufig in verschiedenen Regionen des Nestes, die verschiedensten Mitosenstadien - zwischen Spirem und Telophasen, gleichzeitig angetroffen.

Es ist somit in erster Linie zu verzeichnen, daß der Synchronismus des Teilungsvorganges in identischen Zellen ein viel laxerer ist, als die im ubrigen so verschiedenartigen Prozesse, wie Degeneration und Spermiogenese.

Von viel größerer Bedentung wird aber die Prüfung der Frage sein, $o b$ der beiderseits scharf abgesetzte, mit der ersten Teilung der Ursamenzelle einsetzende und mit Spermienbildung abschließende Lebenscyclus der Samenzellen stets die gleiche Anzahl Zellteilungen bzw. Generationen einschließt?

Diese Frage muß gesondert in zweifacher Beziehung geprüft werden: 1) ob sich alle Zellen innerhalb eines Nestes in dieser Hinsicht identisch verhalten, und 2) ob die Zahl der Generationen der Samenzellen eine für die gegebene Species im allgemeinen konstante Größe ist?

Es sei zunächst nochmals hervorgehoben, daß der geradezu ideelle Synchronismus der spermatogenetischen Vorgänge innerhalb eines Nestes schon von den Reifeerscheinungen der Samenzellen aus datiert. Es länft sowohl die erste als die zweite Reifeteilung stets simultan $\left.{ }^{1}\right)$ in allen Zellen eines Nestes ah, and es gelangen nie gewöhnliche neben heterotypischen Teilungen im selben Nest zur Beobachtung.

Es muß nun geprift werden, ob alle gleichzeitig zur Reife gelangenden Samenzellen auch stets der gleichen Generation angehören?

Die. Entscheidang läßt sich unschwer fuhren und dürfte wohl als bindend betrachtet werden, unter der einzigen, übrigens völlig gesicherten Voraussetzung, daß jedes Nest schon sehr frühzeitig durch eine bindegewebige Follicularkapsel allseitig abgeschlossen und dadurch ein Zellanstausch zwischen benachbarten Nestern unmöglich gemacht wird.

Sollte nun ein derartiges Nest lanter Zellen gleicher Generation

1) Wenn auch nicht streng synchron (vgl. unten). 
enthalten, so muß die Zellenzahl stets $2^{n}$ betragen 1$)$. Ist die Zellenzahl innerhalb eines Nestes nicht $=2^{n}$, liegt auch eine Degeneration einzelner Zellen ohne Hinterlassung jeder Spur außerhalb des Möglichkeitsbereichs, so ist die Schinßfolgerung ganz unabweisbar, daß innerhalb eines Nestes Samenzellen verschiedener Generationen vorliegen.

Wir haben somit nur eine genügende Anzahl von Zählungen vorzunehmen und die Zahlen für sich sprechen zu lassen.

Eine genaue Abzählung eines aus ruhenden Zellen bestehenden Nestes ist aus technischen Gründen kaum ausführbar. Es läßt sich dieselbe nur an Komplexen mitotischer Zellen vornehmen, wobei anch hier eine Anzahl technischer Schwierigkeiten tiberwunden werden muß. Es ist dieses eine langwierige und mühsame Arbeit: jeder Schnitt muß genau mit der Kamera aufgezeichnet werden, und die einzelnen Zellen, die bei einer Schnittdicke von $9 \mu$ meist auf zwei bis drei Schnitte entfallen, auf den benachbarten Skizzen sicher identifizierbar sein. Eine ungünstige Schnittrichtung oder zufällige Überlagerung vereitelt nicht selten die Sicherheit einer mühsamen Zählung.

Eine zweite, nicht minder wichtige Fehlerquelle besteht zuweilen in der Unmöglichkeit, das untersuchte Zellnest in vollständig sicherer Weise von den benachbarten abzugrenzen. Dies geht natürlich ohne jede Schwierigkeit, solange man sich in der Nähe der Mitte des Nestes befindet (vgl. Fig. 3). Nähert man sich dagegen seinem Ende, so erhält sich die scharfe Abgrenzung nur in denjenigen Fällen, wo das Messer die scharfe Kante des Nestes, nicht aber seine breiten Flächen trifft (vgl. Fig. 1 und 2).

Alle diese Umstände erklären zur Gentige, warum nach sorgfältiger Durchmusterung mehrerer Hunderte verschiedenaltriger Nester aus den Hoden von Salamander, Triton und Axolotl nur etwa 40 derselben zur Aufnahme in die Tabelle kamen.

Es zeigte aber gleichzeitig dieses extensive Studium des Materials, daß spermatogoniale Teilungen ausschließlich epidemieartig auftreten and von sporadischen, vereinzelten Teilungen in diesen Fällen nicht die Rede sein kann.

1) Sollte ein Nest ausnahmsweise auch nicht aus einer, sondern aus zwei Ursamenzellen stammen, so ïndert es selbstverständlich nichts an der Sache, da es uns zunächst nicht auf die Feststellung der Gesamtzahl der vorangegangenen Generationen, sondern nur der Gleichheit aller Zellen innerhalb eines Nestes ankommt. Für die Möglichkeit eines mehr als monophyletischen Nestes liegt aber auch nicht der Schein einer Wahrscheinlichkeit vor. 
Untersuchungen über den zeitlichen Faktor der Zellteilung. II. 461

Es sei noch hervorgehoben, daß die unter der Rubrik II $A$ angefuhrten s nicht genau abgrenzbaren * Nester zu einer Kategorie von

Fig. 1.

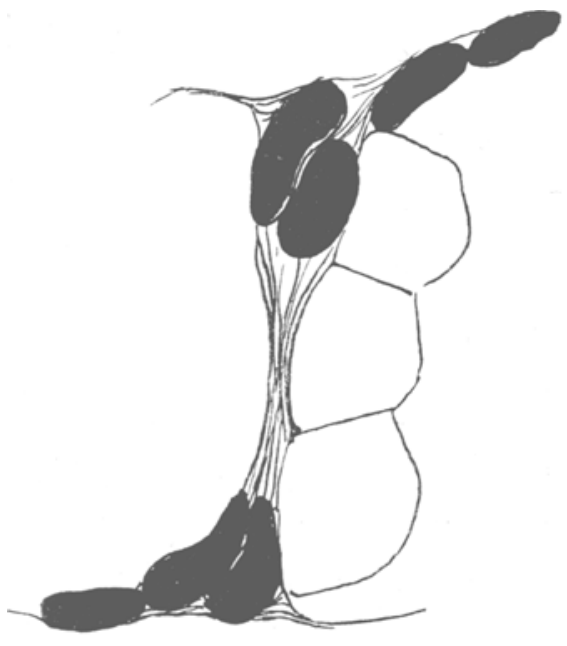

Erster Schnitt
Fig. 2.



Zweiter Schnitt

Fig. 3.

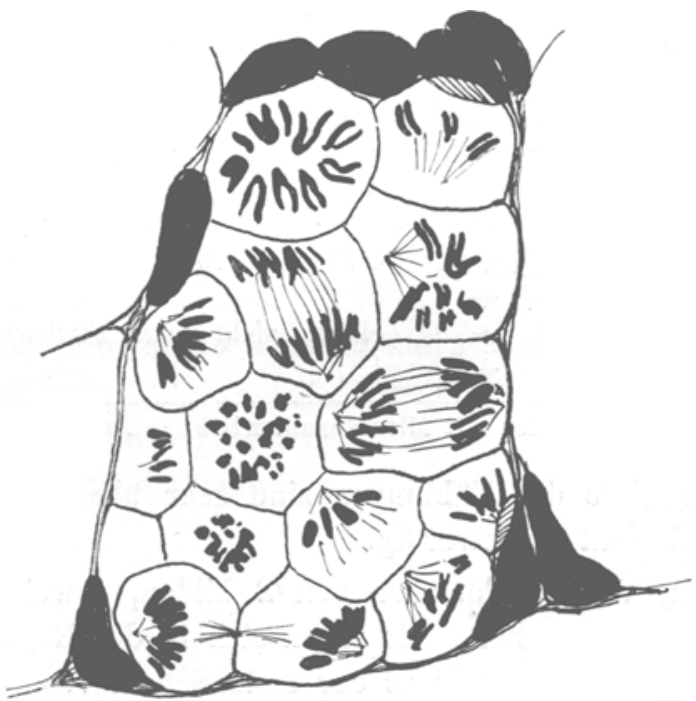

and vierter Schnitt durch ein Nest mit 34 Zellen.

Beobachtungen gehören, wo durch unmittelbare mikroskopische Betrachtung, feines Arbeiten mit der Mikrometerschraube usw. zwar 
eine vollständige Sicherheit der Abgrenzung erreicht wurde, die Beweisfuhrung mittels Skizzen aber nicht gut erbracht werden könnte.

Die sehr zahlreichen Zählungen, in denen eine wenigstens subjektive Sicherheit bezüglich der Zahl der Mitosen oder der Grenzen der Nester nicht erreicht werden konnte, wurden einfach unberücksichtigt gelassen.

Tabelle.

I. Nester mit Mitosenzahlen $=2^{n}$.

\begin{tabular}{|c|c|}
\hline Anzuhl der Nester & Zellenzahl \\
\hline 3 & 4 Mitosen \\
\hline 4 & 8 \\
\hline 6 & 16 \\
\hline 1 & 32 \\
\hline
\end{tabular}

Total: 14 Nester.

II. Nester mit Mitosenzahlen nicht $=2^{n}$.

A. Nester nicht genau abgrenzbar:

Mitosenzahlen: $3,6,7,10-11,13,20-22,20,24-26,39-40$.

B. Genau abgrenzbare Nester:

Mitosenzahlen: 7, 9, 14, 18, 24, 28, 34, 34, 56-58, 1201), 1201), 1141).

C. Nester, in denen neben Mitosen auch ruhende Zellen liegen:

\begin{tabular}{rr|ll}
\hline \hline Anzahl der Mitosen & \multicolumn{2}{|c}{ Ruhezellen } \\
\hline 1 & + & 2 & $=3$ \\
14 & + & 1 & $=15$ \\
12 & + & 4 Paare $=16$ \\
7 & + & $8 ?$ & \\
24 & + & einige (nicht genau zählbar) \\
36 & + & 1 & \\
\hline
\end{tabular}

Total: Nester mit Mitosenzahl nicht $=2^{n}=27$.

Die Ergebnisse der Zählungen sind sehr überraschend und in mehrfacher Hinsicht merkwtirdig.

1) Zahlen, welche Potenzen von 2 bilden, erweisen sich entschieden in der Minderheit. Es teilen sich in der Regel die Zellen nicht so streng simultan, wie es der erste Schein vortäuscht.

1) Erste Reifeteilungen bei Triton. Genaue Zahlen nicht feststellbar, daher die Maximalzahlen angegeben, indem stets jede mit einer benachbarten nieht identifizierbare Zelle als ganze gezählt wurde. 
Untersuchungen über den zeitlichen Faktor der Zellteilung. II. 463

2) Die unter den Rubriken II A und II B angefuhrten Zahlen beweisen, daß wir es mit Zellen verschiedener Generationen zu tnn haben.

Wir wollen für einige Fälle ihre Stammbäume aufstellen, die nur insofern hypothetisch genannt werden dürfen, als sie den einfachsten Hergang zur Voraussetzung haben, $d$. h. mit einem Minimum von Nachzïglern auszukommen suchen (vgl. beistehende Skizzen).

Fig. 4.
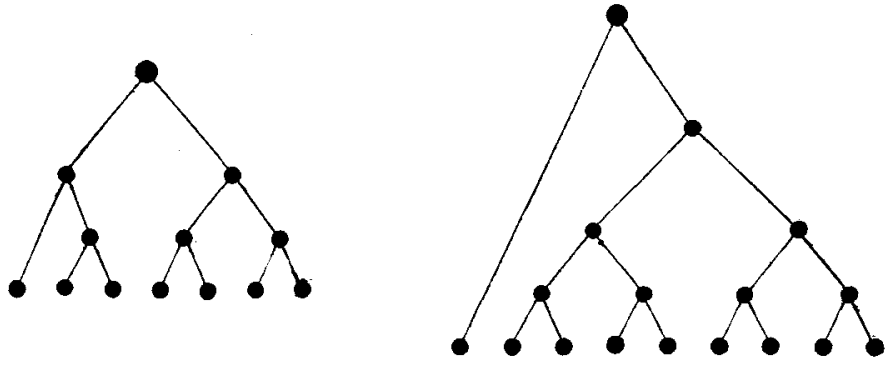

T Zellen

Einfachste Stammbăume von Nestern rait

9 Zellen

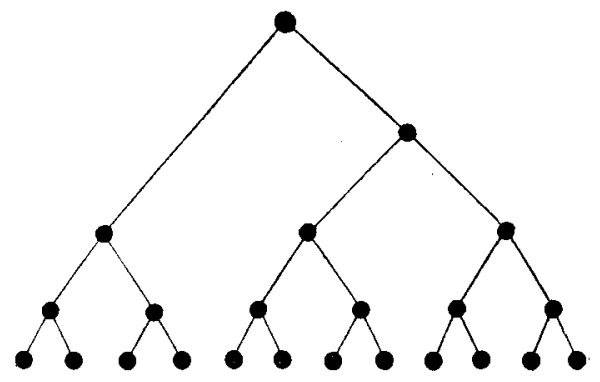

12 Zellen.

a. 7 Zellen können aus einer Zelle am einfachsten folgendermaßen entstehen:

$$
\begin{aligned}
& 7=6+1 \text { Zelle . . . . . . . . . . 3. Generation, } \\
& \text { vorhergehendes Stadium }=3+1 \text { Zelle } . .2 \text {. } \\
& \text { - } \quad-\quad=2 \text { Zellen . . . } 1 . \\
& \text { - } \quad-\quad=1 \text { Zelle . . . . Stammzelle. }
\end{aligned}
$$

Es würden demnach unter 7 Zellen 6 Urenkelzellen und 1 Enkelzelle der Stammzelle sein.

b. 9 Zellen stammen von: $1+4$ Zellen,

$$
\begin{array}{r}
1+2 \\
1+1= \\
1=
\end{array}
$$


c. 24 Zellen stammen von: 12 Zellen,

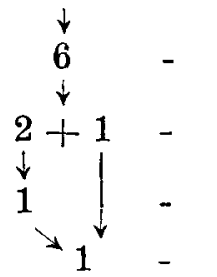

Es wtirden demnach in der Ascendenz einer, wenn auch geraden Zahl, die nicht eine Potenz von 2 ist, stets mindestens ein, vielfach auch mehrere Ausfälle von Teilungen in vereinzelten oder ev. auch mehreren Zellen anzunehmen sein.

3) Die ausgefallenen Teilungen werden nicht mehr oder nur zum Teil nachgeholt.

Es erhellt dies schon ans dem Fehlen sporadischer Teilungen, wird aber des weiteren durch die Ergebnísse der Zäblungen von heterotypischen Teilungen bekräftigt. Derartige Nester enthalten in allen gezählten Fällen maximum 120 Zellen, eine Zahl, die $2^{7}=128$ nahe kommt 1 ).

Wir können daraus schließen, daß die 1., heterotypische Reifeteilung in der Regel in die 7. Generation der spermatogonialen Zellen fällt, daß aber daneben stets auch einige oder mehrere Zellen früherer Generationen zu Spermatocyten werden.

Prüfen wir nun der Reihe nach die sich ergebenden Konsequenzen.

Wenn wir die Nester mit geraden Zahlen, die nicht zugleich Potenzen von 2 sind, beruicksichtigen, so sehen wir, daß eine Zelle, die an einer bestimmten Teilungsepidemie nicht beteiligt war, sich noch nachträglich, und zwar stets gleichzeitig mit den übrigen Zellen einer ihr bereits fremden Generation, eventuell auch wiederholt, teilt.

So kann z. B. die Zahl 14 usw. nur dadurch entstehen, daß gleichzeitig mit 6 Zellen dritter Generation sich auch eine Zelle zweiter Generation an der Teilungsepidemie beteiligt. Zahlen wie 28, 56, usw. legen sogar die Annahme am nächsten, daß der frühere Nachzügler nunmehr in Reih und Glied mit dem Gros der Zellen mehrere Teilungen durchmacht.

1) Abgesehen von den drei gezählten Nestern mit heterotypischer Teilung warden noch 6 Bündel reifender Spermien gezühlt, die stets die Zahl $>480$ und $<512=2^{9}$ ergaben. 
Es sind in diesem Ergebnis mehrere Umstände gleich auffallend und bedeutend:

a. Der Ausfall einer Teilung kann nunmehr nicht etwa so aufgefaßt werden, daß die betreffende Zelle einfach untauglich zur Teilung geworden ist, da sie sich ja nachträglich wieder teilt.

b. Handelte es sich nur um ein Versäumnis seitens der Zelle, um ein ausnahmsweise hohes Schwankungsmaß ihres Teilungsrhythmus, so ist ja gar nicht einzusehen, warum nicht die versänmte Teilung nachträglich unabhängig von den übrigen Zellen erfolgt, warum mit andern Worten Zellen verschiedener Generationen sich stets simultan teilen.

Hat die Mehrzahl der Zellen des betreffenden Nestes die kritische siebente Generation erreicht, die das Eintreten in die Reifungsperiode bedeutet, so machen auch diejenigen Zellen die heterotypische Teilung mit ihren Konsequenzen durch, welche an sich genommen dazu gewissermaßen gar nicht berechtigt wären, da sie einer früheren Generation angehören. Ist aber das Eintreten in die heterotypische Teilung an sich nicht an eine bestimmte Generation gebunden, warum werden derartige Teilungen nie sporadisch, mit Teilungen somatischen Charakters untermengt, vorgefunden?

Es sind, wie wir sehen, lauter Widersprüche, Antithesen, die uns auf den ersten Blick ganz stutzig machen müssen und deren befriedigende Lösung einen offenen Bruch mit so manchen Überlieferungen der Cytologie erheischt.

Wenn wir, um unsre Betrachtungen einfacher und durchsichtiger zu gestalten, von der höchst unwahrscheinlichen Annahme einer von außen induzierten Zeitbestimmung für die Reifeprozesse und den Beginn der Spermatogenese absehen, so müssen wir den Sachverhalt folgendermaßen ausdrticken:

In der Nachkommenschaft jeder Ursamenzelle laufen streng synchron bestimmte Prozesse ab, deren Abschluß wir in der Spermatogenese, deren notwendiges Zwischenstadium in den Reifeteilungen erblicken.

Der Ablauf derselben wird durch die dazwischenfallenden Zellteilungen weder gehemmt, noch gefördert, ist vielmehr von denselben völlig unabhängig; eine vollzogene Zellteilung ist in diesen Falle nicht mit einem Entwicklungsschritt gleichbedeutend. Es folgt letztere Aussage unmittelbar ans der oben erschlossenen synchronen Reifeteilung und der spermatogenetischen Entwicklung von Samenzellen verschiedener Generationen. 
Eine Zelle schreitet zur heterotypischen Reifeteilung nicht, weil sie zur siebenten Generation, von der Ursamenzelle aus gerechnet, gehört, sondern weil in ihr bestimmte Prozesse als abgelaufen gedacht werden müssen, die - unter den gegebenen Lebensbedingungen bei allen Zellen des Nestes eine bestimmte gleiche Zeitspanne beanspruchen.

Diese Konsequenz steht in einem denkbar schroffen Gegensatz zu einer bereits älteren, aber neuerdings bekräftigten Aussage Boveris.

In bezug auf die Spermatogenese bat sich letzterer Autor folgendermaßen geäußert: $\gg$ An jede (Zell-) Teilung ist ein gewisses Quantum von Veränderung geknüpft, so daß jede folgende (Zell-) Generation dem zu erreichenden Ziel, dem Spermatozoon, wieder um einen Schritt näher gerückt ist. $\mathrm{DaB}$ diese Veränderungen gerade an die Teilung geknüpft sein müssen, läßt sich leicht einsehen; denn würden sie schon früher sich ausbilden, würde z. B. die Spermamutterzelle schon vor der Teilung die Eigenschaften des Spermatozoons annehmen, dann wäre sie eben ein Spermatozoon und würde sich nicht mehr teilen ....11).

Es kann aber ja gleichzeitig nicht außer acht gelassen werden, daß, wenn nicht das Minimnm, so doch allem Anscheine nach das Maximum der spermatogonialen Teilungen, und zwar auf 6 festgesetzt ist, daß dieselben nur epidemieweise auftreten, und daß eine ausgefallene Teilung nur bei der nächsten Epidemie nachgeholt werden kann. Die Gesamtheit dieser Umstände läßt sich nur in dem Sinne interpretieren, daß jeder Samenzelle während ihres Entwicklungscyclus sechsmal die Möglichkeit zur Teilung, und zwar in bestimmten, durch irgendwelche innere Vorgänge fixierten Zeitabständen geboten wird. Es kann aber die eine oder die andre Zelle eine oder mehrere Gelegenheiten an sich unverwertet vorbeiziehen lassen, ohne dadurch aus der normalen Entwicklungsbahn auszuscheiden.

Diese Feststellung scheint uns in zweifacher Hinsicht von fundamentaler Bedeutung.

1) Es ergibt sich in erster Linie, daß für identische Zellen ibre Identität nicht nur aus ibrer Organisation, sondern auch aus der Gesamtheit ihrer Lebensäußerungen erschlossen - in bezug auf den Teilungsakt nur eine Gleichheit der Möglichkeiten,

1) Die Potenzen der Asearis-Blastomeren. Festschr. f. R. HeRTwig. Bd. III. S. 194. (Selbstzitat einer Arbeit aus dem Jahre 1886.) 
nicht der Verwirklichung derselben besteht. Wir finden darin eine Bestätigung unsrer Ansicht, daß dem Teilungsvorgang eine Sonderstellung in der Reihe der übrigen Lebensprozesse der Zelle eingeränmt werden muß, insofern als derselbe, vielleicht abgesehen von einigen weiter zu erörternden Fällen, nicht in fataler Weise durch die Gesamtheit der Lebensumstände der Zelle prädeterminiert werden kann.

2) Es tritt uns der Begriff der $\star$ Teilungsmöglichkeit* als ein konkreter, weiter analysierbarer und erkennbarer Faktor entgegen.

Es ist in der Tat eine ganz unerwartete und bedeutungsvolle Tatsache, daß die Wahrscheinlichkeit des Eintreffens einer Mitose nicht in Abhängigkeit von dem Zeitabstande von der letzten stattgefundenen zunimmt: eine Spermatogonie, die bei der letzten vorangegangenen Teilungsepidemie sich an derselben nicht beteiligte, gewinnt dadurch keinen Vorsprung den juingeren, eben aus der Teilung hervorgegangenen Zellen gegenüber. Es wächst vielmehr in derselben, streng synchron mit den vibrigen Zellen jüngerer Generation, die Möglichkeit für den nächsten Teilungsschritt heran.

Wir können uns, auf Grund des Ausgeführten, eine folgende Vorstellung von dem Wesen des Möglichkeitsfaktors der Teilung bilden :

Beim Zustandekommen der Zellteilung gehört ein Faktor zur Voranssetzung, dessen folgende Eigenschaften uns nunmehr bekannt sind:

a. Er tritt periodisch auf.

b. Die Zahl der Perioden ist (möglicherweise nur in bestimmten Zellarten) genau festgesetzt.

c. Die Periodizität dieses Faktors, bzw. die derselben zugrunde liegenden Prozesse, sind von einer ev. Verwirklichung oder Nichtverwirklichung der durch denselben gebotenen Teilungsmöglichkeit unabhängig.

d. Es ist sehr wahrscheinlich, obwohl erst zu beweisen, daß in identischen Zellen auch die Perioden des Faktors übereinstimmen, was unter Voraussetzung der Gleichheit der sonstigen Lebensbedingungen der betreffenden Zellen, zum synchronen Auftreten desselben in allen Zellen fuhren muß.

Die unter $c$ angefuhrte Konsequenz erscheint mir besonders bemerkenswert, da sie im schroffen Gegensatz zu der allgemeinen Anschauung über die Bestimmung des Zeitpunktes der Zellteilung steht. Denken wir an die Fassung von Boveri, von R. Hertwig, oder eine ähnliche, so dominiert überall die Vorstellung einer allmäh- 
lichen Heranreifung eines Zustandes in der Zelle, welcher schließlich in der Teilung derselben gipfelt, um unmittelbar $\mathrm{n}$ ach der Teilung anf sein tiefstes Niveau herabzusinken. Die Periodizität wird hier erst durch das Stattfinden der Teilung hervorgebracht, wogegen der Möglichkeitsfaktor, dessen Aufstellung sich uns hier aufdrängt, unbekümmert um die tatsächlich vollzogene Teilung periodisch auftritt. In dieser seiner Unabhängigkeit von den mächtigen, durch die Mitose in der Zelle erzeugten Umwälzungen liegt seine wichtige, wenn auch vorderhand negative Charakteristik, die uns Ausblicke auf eine tiefergehende Analyse desselben eröffnet.

Wir dürfen es natürlich nicht unterlassen, uns volle Klarheit darüber zu verschaffen, inwiefern in unsrer vorangehenden Konstruktion hypothetische Elemente eingeschlossen sind.

Der Begriff der * Möglichkeit* scheint auf den ersten Blick manche Berührungspunkte mit Driescress »prospektiver Potenz zu besitzen. Es handelt sich jedoch um grundverschiedene Dinge: Drieschs und andrer Autoren experimentelle Ermittlungen zeigen, daß bestimmte Organe oder sonstige Körperelemente anderes und mehr können, als sie unter normalen Umständen Ieisten.

Wir behaupten dagegen von unserm Objekte, das unter gegebenen Umständen in bewußtem Sinne nichts leistet, daß es, und zwar nur jetzt, im gegebenen Zeitpunkte ein Bestimmtes vollbringen könnte; and setzen selbstrerständlich stillschweigend ein *wenn * hinza.

In diesem *Wenn gipfelt der Schwerpunkt unsrer Untersuchung und zugleich ihr hypothetisches Element.

Von der Tatsache der unleugbaren und vollständigen Identität der betreffenden Zelle mit den übrigen Elementen des Nestes, und zwar hinsichtlich ihrer Herkunft, mannigfaltiger physiologischer $\mathrm{Zu}$ stände (Degeneration!) und prospektiver Bedeutung ausgehend, machen wir die, allerdings hypothetische Annahme, daß das beim Wegfall einer Mitose sich offenbarende abweichende Verhalten der einen oder der andern Zelle nicht auf einer nomologischen, sondern einer bloB ontologischen Verschiedenheit derselben beruht, daB mit andern Worten auch in dieser Zelle die Gesamtheit der für die Teilung notwendigen Umstände, bis auf bestimmte Beziehungen zu irgendeinem Faktor, vorliegt, wobei letztere Beziehungen eben nicht zur nomologischen Bestimmung der betreffenden Zellenart gehören.

Diese »Gesamtheit der Umstände*, die nachweisbar periodisch auftritt, bezeichnen wir eben als $\gg$ Möglichkeit . 
Wollten wir die Realexistenz derselben negieren, so hieße es mit andern Worten, daß eine Zelle, die bis dahin und nachher wieder in ihrem Verhalten mit ihren Schwesterzellen vollständig tubereinstimmte, während der epidemischen Teilung, an der sie sich nicht beteiligte, in einem vollständig indifferenten Zustande verblieb, d. h. daB die ganze, wohl höchst komplexe Gesamtheit der die Mitose bedingenden Umstände, derselben vollständig abkam. Daß diese »mitotischen Momente « mit dem ganzen Wesen der Zellen eng verbunden sind, zu ihrer nomologischen Bestimmung gehören, erhellt aber gleichzeitig aus der Periodizität der Teilungen und dem genau geregelten Maximum der Perioden.

Wir glauben demnach, mit der Realexistenz eines als $>$ Teilungsmöglichkeit^ aufzufassenden Zustandes als mit einer wohlverbürgten Tatsache rechnen zu dürfen.

In diesem »Möglichkeitsfaktor *, welcher für das Zustandekommen des Teilungsvorganges noch einen "Verwirklichungsfaktors zur Voraussetzung hat, erkennen wir unsre ursprüngliche Fiktion zweier unabhängiger Ursachen (richtiger Ursachengruppen) wieder, von der wir bei der Aufstellung des Begriffes der Normierung ausgingen. An Stelle einer ganz leeren Hypothese über das Wesen dieser Faktoren konnten wir nunmehr im vorhergehenden einige konkrete Angaben über die Eigenschaften des einen Faktors machen.

Wir wollen nun der Analyse des zweiten Faktors etwas näher treten.

Es sei vorweggenommen, daß die spermatogonialen und Reifeteilungen in vollem Maße unsrer Definition der Determination des zeitlichen Faktors entsprechen: die Teilungen erfolgen zur bestimmten Zeit oder uberhaupt nicht.

Es soll nun untersucht werden, ob wir Anhaltspunkte für eine nähere Zuweisung derselben zu einer unsrer vorher aufgestellten Kategorien der Determination gewinnen können.

Es kommen hier zwei Punkte in Betracht.

1) Der mangelhafte Synchronismus der Mitosenstadien innerhalb eines Nestes.

Es wurde bereits hervorgehoben, daß wir nur selten Nester antreffen, in denen nicht verschiedene, und zwar weit abstehende Mitosenstadien anzutreffen wären. Abgesehen von den jüngeren Generationen, von 4-8 Zellen, wo nicht selten ein recht befriedigender Synchronismus zu beobachten ist, habe ich nur einen Fall der späteren Generationen zu verzeichnen, wo auf 109 (heterotypische) 
Äquatorialplatten ein einziges abweichendes Stadium, und zwar eine Anaphase, entfiel.

Es läßt sich der Nachweis erbringen, daß diese mangelhafte zeitliche Koinzidenz der Mitosenstadien nicht auf die verschiedenen Tempi der einzelnen Abläufe beruht, da sie sich schon in den allerfrühesten Stadien der Mitose bemerkbar macht. Es gewinnt daher dieser Umstand, namentlich wenn man denselben dem geradezu ideellen Synchronismus sowohl der Degenerationsvorgänge als der spermatogenetischen Prozesse gegeniiberstellt, eine prinzipielle Bedeutung. Dieselbe wird noch dadurch erhöht, daß

2) Ein ganz unverkennbares Voraneilen der dem Cystenlumen näher liegenden Zellen im Vergleich zu den mehr peripheren besteht. Es ist dieses zwar kein strenges Gesetz, aber eine sehr häufig, zuweilen in ganz exquisiter Form wiederkehrende Erscheinung, welche besonders bedeutsam wird, wenn man sie mit einigen, auf verschiedene Syncytien bezüglichen Tatsachen zusammenstellt.

Es liegen einige in der Literatur zerstrente Angaben über Mitosen in normalen und pathologischen Syncytien vor, welche neben weiteren diesbezüglichen Beobachtungen von einer meiner Schülerinnen demnächst mitgeteilt werden sollen. Es scheint, daß die Teilungen in diesen Gebilden so gut wie ausnahmslos synchron verlaufen, daneben aber, namentlich bei größerer Ausdehnung des plasmatischen Feldes, ein gewisses Fortschreiten des Vorganges in einer bestimmten Richtung wahrnehmbar ist. Ich will hier nur an die berühnte ältere Figur von Strassburger erinnern, wo in einem breiten Syncytialstreifen aus der Embryosackbekleidung von Fritillaria ganz streng zonenweise der mitotische Vorgang von den Stadien der Prophasen bis an die Telophasen fortschreitet.

Diese Beobachtungen, die natürlich vervielfältigt und vertieft werden müssen, sind von unserm Standpunkte in dem Sinne zu deuten, daß das Zusammentreffen unsres Möglichkeits- und Verwirklichungsfaktors ein durch den ganzen Zellkomplex gewissermaßen wellenförmig fortschreitender Vorgang ist. $\mathrm{Da}$ wir anderseits, wie oben dargelegt, annehmen müssen, daß der eine der Faktoren (vorher als Möglichkeitsfaktor « bezeichnet) im ganzen Komplex streng synchron ist, haben wir das vorhin verzeichnete Fortschreiten des Prozesses in bestimmter Richtung dem zweiten, dem »Verwirklichungsfaktor*, auf Rechnung zu setzen. Es ist demnach letzterer gewissermaßen als „Kollektivfaktor" aufzufassen, indem er als ein gewisser Zustand sich durch den ganzen Komplex zonenweise fort- 
Untersuchungen über den zeitlichen Faktor der Zellteilung. II. 471

pflanzt und alle vorliegenden Individuen, sc. Zellen oder (in Syncytien) Kerne, ergreift.

Etwas Näheres über denselben zurzeit auszusagen, hieße ganz vage und unbegrïndete Hypothesen erfinden.

Wenn wir uns auf Grund des Ausgeführten eine Vorstellung über die Art der in unserm Objekte verlaufenden Determination bilden wollen, so scheint es wohl am nächstliegenden, den Sachverhalt etwa folgendermaßen zu deuten.

a. Der Verwirklichungsfaktor * ist (rom Standpunkt des ganzen Zellkomplexes betrachtet) stets zur nötigen Zeit, d. h. während der entsprechenden Periode des "Möglichkeitsfaktors", in Tätigkeit. Ob es sich um eine funktionale Beziehung zwischen beiden Faktoren handelt, wie solche in meinem Schema als 1) vorgesehen ist, oder ob hier eine konstante, unveränderliche *Bedingung* des Komplexes vorliegt, muß zunächst unentschieden bleiben.

b. Wäre nun der "Möglichkeitsfaktor *, welcher hier mit der Gesamtheit der Zustände jeder Zelle identisch ist, sowohl in nomologischer, wie in ontologischer Hinsicht in allen Zellen identisch, so müßten die Mitosen zur bestimmten Zeit ganz ausnahmslos eintreffen.

Angesichts der tatsächlichen Verhältnisse, d. h. des nicht seltenen Ausbleibens derselben, bleibt uns nur die Annahme übrig, daß irgendein Parameter des Möglichkeitsfaktors unbestimmt bleibt. Daß diese Zulassung dem Tatbestande entspricht, erhellt schon darans, daB die räumliche Orientierung der mitotischen Figuren im Neste keine gesetzmäßige ist.

Die identischen Zellen sind somit nicht in identischer Weise in bezug auf den gemeinsamen Verwirklichungsfaktor orientiert und dieser Umstand mag gentigen, um uns die Art der Determination verständlich zu machen. 